\title{
Investigation of Radiated Emissions of a Galvanically Isolated qZS DC-DC Converter
}

\author{
Tanel Jalakas ${ }^{1}$, Marek Jarkovoi ${ }^{1}$, Indrek Roasto ${ }^{2}$, Janis Zakis ${ }^{3}$, Alexander Garganeev ${ }^{4}$ \\ ${ }^{1}$ Tallinn University of Technology, Tallinn, Estonia \\ ${ }^{2}$ Ubik Solutions, Tallinn, Estonia \\ ${ }^{3}$ Riga Technical University, Riga, Latvia \\ ${ }^{4}$ National Research Tomsk Polytechnic University, Tomsk, Russia \\ tanel.jalakas@ttu.ee
}

\begin{abstract}
Ten different modulation methods of the isolated quasi $\mathrm{Z}$-source inverter based DC-DC converter were tested to find out radiated emission levels. EMC measurements were performed on a low power $250 \mathrm{~W}$ prototype. A radiated emission test was carried out in a GTEM cell (gigahertz transverse electromagnetic cell) according to the EMC standard IEC 610004-20. To obtain a complete overview of the emissions, both $C$ and $D$ bands were included in a single test at the start and stop frequencies $30 \mathrm{MHz}$ and $1 \mathrm{GHz}$, respectively. Measuring bandwidth of $120 \mathrm{kHz}$ with a $6 \mathrm{~dB}$ EMI filter was used.
\end{abstract}

Keywords-Quasi $Z$ source, DC-DC converters, isolated boost converters, radiated EMI measurement

\section{INTRODUCTION}

The object of the study is a low power DC-DC converter stage for PV microinverters. The technology in PV Microinverters is moving in direction of increasing power density, which means smaller dimensions and lighter casing. Conventional microinverters have metallic casing with good EMI shielding properties. One alternative approach is to use nonmetallic casing that allows it to increase the power density and lower the price. However, in the case of nonmetallic casing radiated emission could cause EMI issues.

Radiated emission of power electronics converters is influenced by a variety of factors, for instance, topology of power circuit, PCB design, component properties, parasitic properties, shielding/casing and power electronics modulation methods. Different modulation methods have direct effect on the converter's EMC properties as well on the efficiency and its voltage boost ability. In the current paper radiated emission of the voltage boosting quasi Z-source inverter (qZSI) [1], [2] based isolated DC-DC converter is measured. Different modulation methods are compared to find out the modulation method that emits least radiated EMI. This is the first step in a series of EMI investigations with the goal to study EMC properties of the qZSI based isolated DC-DC converter.

\section{OBJECT OF INVESTIGATION}

The object of the study is a low power DC-DC converter stage for PV microinverters (Figure 1). The purpose of the developed converter is to stabilize and step up the fluctuating PV panel output voltage. Another important task of this converter is to provide galvanic isolation between the microinverter low voltage primary and the high voltage secondary side. The first stage of this converter is based on the qZSI. Voltage is boosted by inductors L1 and L2. Voltage boost is controlled by the inverter's shoot-through cycles. To store energy to L1 and L2 during the shoot-through cycle, one or both inverter arms will be short circuited. The second stage is a high frequency $(100 \mathrm{kHz})$ step-up (1/4) transformer. The third stage is a voltage doubler rectifier.

The advantages of the Z-source converter family as well as its basic control and operation principles are described in [1], [2], [3]. The test parameters are shown in Table 1 and the experimental prototype in Figure 2.

TABLE 1 TEST PARAMETERS

\begin{tabular}{|l|c|}
\hline \multicolumn{1}{|c|}{ Parameter } & Value \\
\hline Min. input voltage $U_{\text {in min }},(\mathrm{V}$ DC) & 15 \\
\hline Max. input voltage $U_{\text {in } \max },(\mathrm{V}$ DC) & 18.5 \\
\hline Output voltage $U_{\text {out }},(\mathrm{V}$ DC) & 230 \\
\hline Output load $R_{\text {out }},(\Omega)$ & 600 \\
\hline Output power $P_{\text {out }},(\mathrm{W})$ & 88 \\
\hline Switching frequency $f_{\text {sw }},(\mathrm{kHz})$ & 100 \\
\hline
\end{tabular}

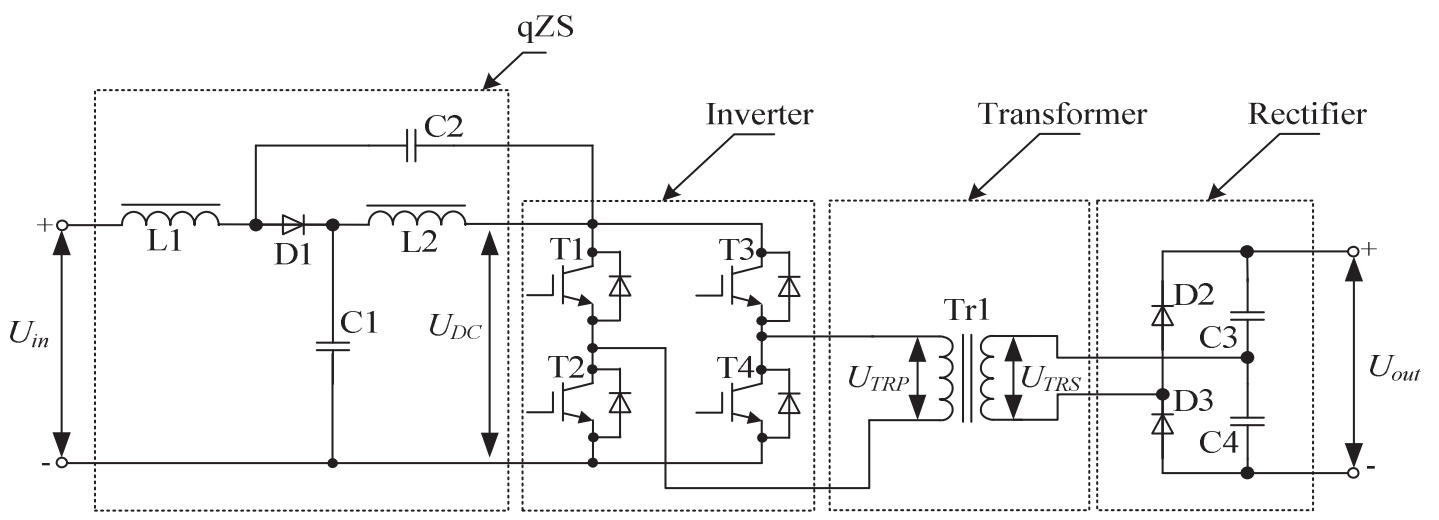

Figure 1 Isolated qZS inverter based DC-DC converter. 


\begin{tabular}{|l|c|}
\hline Shoot-through duty cycle $D_{s},(\%)$ & 25 \\
\hline $\mathrm{L} 1$ and $\mathrm{L} 2$ inductances $L_{1}, L_{2},(\mu \mathrm{H})$ & 11 \\
\hline $\mathrm{C} 1$ and $\mathrm{C} 2$ capacitance $C_{l}, C_{2},(\mu \mathrm{F})$ & 26.4 \\
\hline Transformer transfer ratio $N_{p} / N_{s}$ & $1 / 4$ \\
\hline Input diode $D_{1}$ & V60D100C \\
\hline Transistors $T_{1}, T_{2}, T_{3}, T_{4}$ & Si4190ADY \\
\hline Rectifier diodes $D_{2}, D_{3}$ & C3D08060A \\
\hline C3 and C4 capacitance $C_{3}, C_{4},(\mu \mathrm{F})$ & 2.2 \\
\hline
\end{tabular}

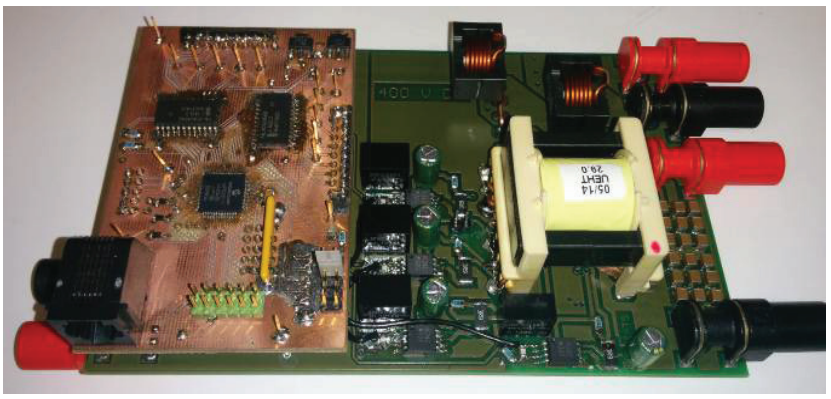

Figure 2 Isolated DC-DC converter prototype.

During the tests the output voltage and the power were kept constant. Only the input voltage was regulated to compensate the different voltage boost properties of the investigated modulation methods.

\section{Tested Shoot-Through Modulation Methods}

Ten different modulation methods were tested: Method A...Method J. During the tests, EMC properties were measured with the GTEM cell and a spectrum analyzer. However, it must be noticed that besides the radiated emission, also the efficiency of the converter depends on the modulation method.

\section{A. PWM Control with Shoot-Through During Zero States (Method A)}

The PWM control with shoot-through during zero states is the most common modulation method of a qZS-based DC-DC converter. Not to disturb the shape of the primary voltage of the isolation transformer, shoot-through states are placed inside zero states, as shown in Figure 3. As a result, the number of higher harmonics will be minimized. It must be pointed out that zero states are always generated by the same pair of switches: either the top (T1 and T3) or the bottom (T2 and T4) transistors. Currently, the top side transistors were used. During the shoot-through, all four transistors are conducting.

\section{B. PWM Control with Shifted Shoot-Through (Method B)}

Method B was introduced in [4] as an improved alternative to the classical PWM shoot-through modulation method (Method A). The number of shoot-through states per period is limited by two. Previous tests have shown that shoot-through in the middle of zero vector will cause more overvoltage peaks as compared to methods without zero vector. To minimize such peaks and reduce radiated emission, one shoot-through state is shifted towards an active state until they merge, as shown in Figure 3. As a result, soft switching ZVS is achieved for the bottom transistors (T2, T4). The shoot-through states are generated directly after active states. During the shoot-through, all four transistors are conducting. Zero states come after shoot-through states. Thus, with this modulation technique, lower EMI is expected.

\section{PWM Control with Shifted Shoot-Through in One Leg (Method C)}

This modulation method is derived from Method $B$. Unlike Method B, here only two switches of one leg are used at a time, as shown in Figure 3. The shoot-through is generated after active states. As a result, the overvoltage peaks appearing on transistors were reduced, which should result in lower EMI compared to Method B. On the other hand, the amplitude value of the shoot-through current is increased, since the number of simultaneously conducting shoot-through transistors has been reduced. In the end, this leads to higher conduction losses during the shoot-through state.

\section{Asymmetric PWM Control with Shifted Shoot-Through in One Leg (Method D)}

Method D derrived from Method $C$ is characterized by the asymmetric transformer voltage. Since shoot-through within

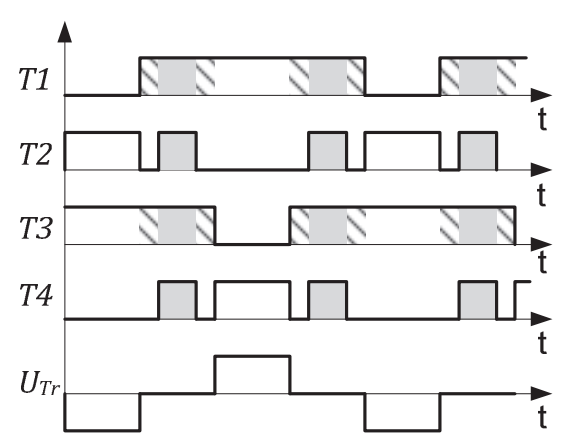

(Method A)

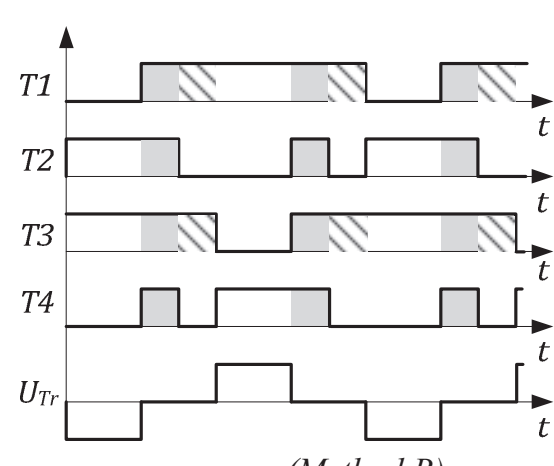

(Method B)

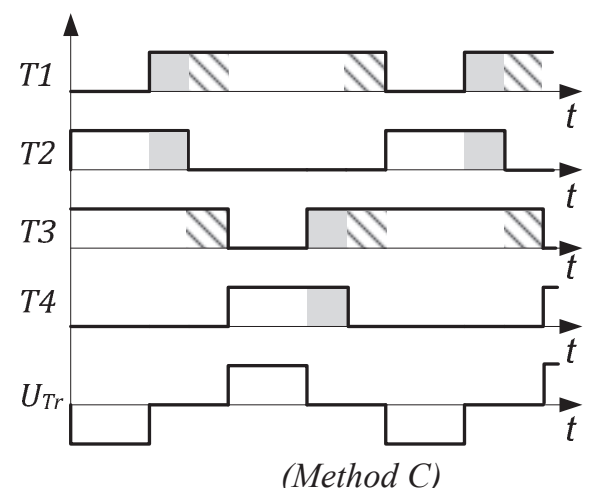

(Method C) 


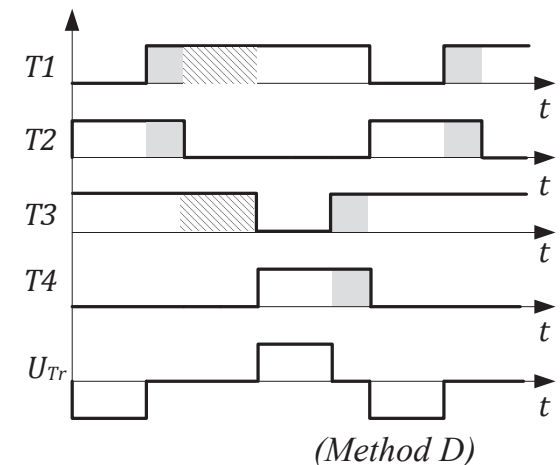

(Method D)

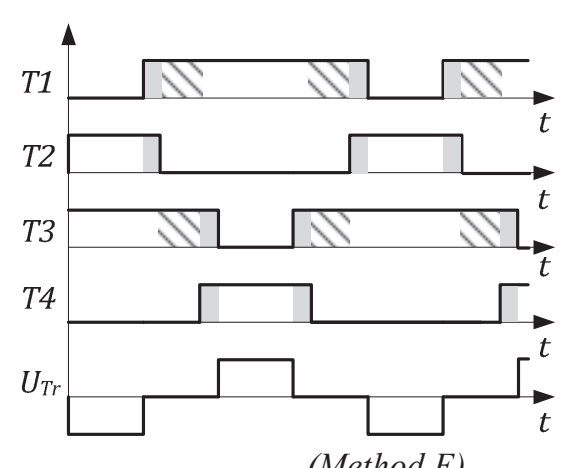

(Method E)

Figure 4 PWM shoot-through modulation methods.

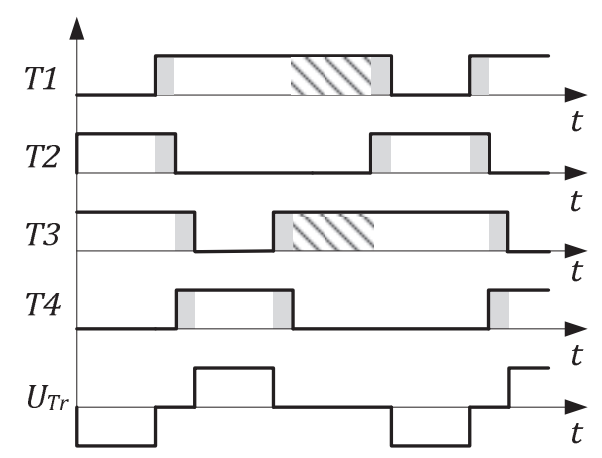

(Method F) zero states tends to create higher overvoltage peaks, it is favorable to eliminate zero states. In Method $D$ this goal is partly achieved by shifting active states towards each other so that one of the zero states could be eliminated (Figure 4). As a result, the EMI emission should be smaller than in Method $C$. However, the asymmetric isolation transformer voltage and the changing frequency of shoot-through states in Method D affect the input current and the output voltage ripple. During the shoot-through, two transistors are conducting.

\section{E. PWM Control with Shifted Double Shoot-Through in One Leg (Method E)}

This modulation technique is a derivation from Method $C$. The shoot-through states are split in two and positioned on both sides of the active states (Figure 4). The shoot-through current is distributed between two transistors at a time. Due to the increased number of shoot-through states, this method is expected to have higher levels of radiated emission.

\section{F. Asymmetric PWM Control with Shifted Double Shoot- Through in One Leg (Method F)}

The asymmetric PWM control with shifted double shoot-through in one leg was derived from Method E. The basic idea behind Method $F$ is to shift active and shoot-through states towards each other so that one of the two zero states could be eliminated, as shown in Figure 4. As a result, as compared to Method E, lower levels of radiated emission will be expected.

\section{G. PWM Control with Shoot-Through During Free-wheeling States (Method G)}

It is also possible to generate shoot-through states during freewheeling states where all the inverter switches are turned off (Figure 5). During the shoot-through, all four transistors are conducting. The switching frequency of all the transistors is equal. In [5] overvoltage spikes across the transistors were noticed, which could cause additional EMI. They are caused by the leakage energy stored in the isolation transformer.

\section{H. PWM Control with Shoot-Through by the Overlap of Active States (Method H)}

The shoot-through is achieved by increasing the duty cycle of active states over 0.5 . This causes the active states of bottom $(T 2, T 4)$ and top $(T 1, T 3)$ side transistors to overlap, as shown in Figure 5. During the shoot-through mode, all four transistors of the qZS inverter are conducting and the current through the inverter switches reaches its maximum. The

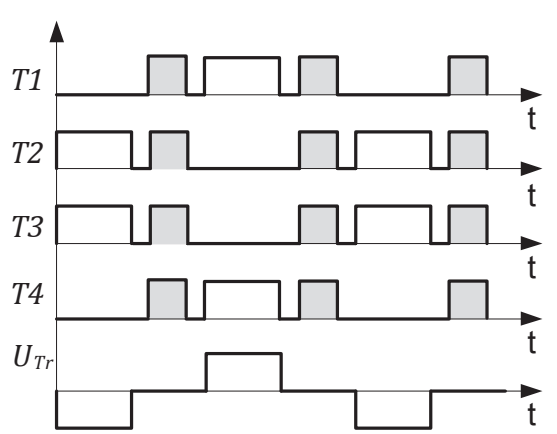

(Method G)

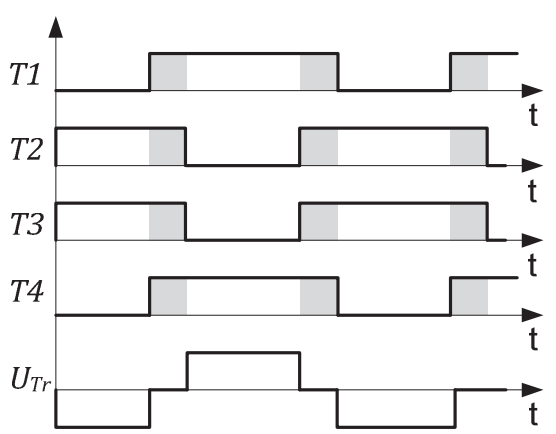

(Method H)

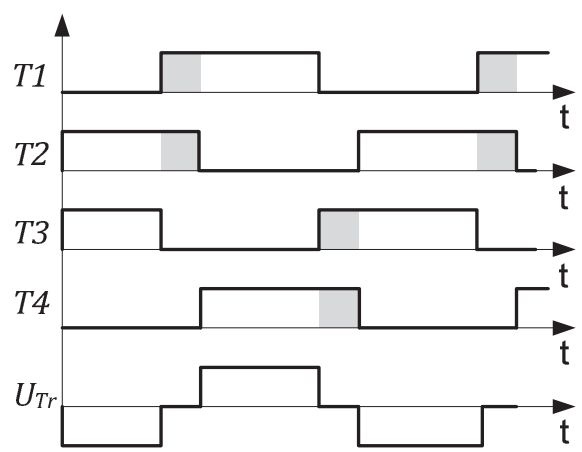

(Method I)

Figure 5 PWM shoot-through modulation methods. 
operating period of the isolation transformer in this modulation method consists of a shoot-through state and an active state. Missing zero states should reduce radiated emission. In principle, Methods $G$ and $H$ are very similar and probably also from the EMI point of view.

\section{PWM Control With Shoot-Through by the Overlap Of Active States in Single Leg (Method I)}

According to the symmetrical overlap principle, the shoot-through states are generated by increasing the duty cycle of active states. The idea is to create shoot-through by shifting the active states towards each other while keeping the duty cycle constant at 0.5 . Figure 5 indicates the shoot-through generation principle of Method I. During shoot-through, only two switches at the time are conducting.

\section{J. PSM control with Shoot-Through During Zero States $(\operatorname{Method} J)$}

An alternative to PWM is the phase shift modulation (PSM) shoot-through control. Figure 6 shows the PSM principle of a single-phase qZS inverter where shoot-through is generated during zero states. The active states are controlled with the phase shift between the control signals. Unlike Method A where zero state was always generated by the same pair of transistors (T1/T3 or T2/T4), here the pairs are alternating twice in each period. As a result, the transistors are equally loaded. However, from the EMI perspective, Methods $A$ and $J$ are similar.

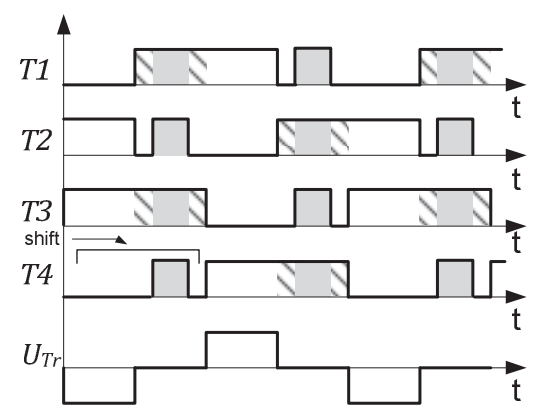

Shoot-through states

Zero states

Figure 6 PSM shoot-through modulation method J.

\section{EMC MEASUREMENTS}

To compare emission levels of different modulation methods, EMC measurements were performed on the DC-DC power converter. Radiated emission test was carried out in a GTEM cell (gigahertz transverse electromagnetic cell) according to the EMC standard IEC 61000-4-20 [6]. Voltage at the RF port of the GTEM cell was measured in $\mathrm{dB} \mu \mathrm{V}$ using a Rohde \& Schwartz ESL6 EMI test receiver at three different orientations of the DC-DC converter board for each modulation method. The voltage was then correlated to $10 \mathrm{~m}$ open air test site (OATS) electric field according to the standard one port transverse electromagnetic (TEM) waveguide correlation algorithm. The OATS electric field data can be used to compare radiated emission levels and evaluate emissions against product-specific standards.

The standard one-port TEM waveguide correlation algorithm used in the test consists of the following steps:

1. Calculating the vector sum of the three measured voltages at different orientations of equipment under test (EUT);

2. Determining the TEM mode field factor using an experimental or analytical procedure (calculation);

3. Calculating the total radiated power emitted by the EUT;

4. Calculating the geometry factors of an equivalent dipole for horizontal and vertical polarization at specified OATS configuration;

5. Calculating an estimate for the maximum field strength on an OATS using the maximum geometry factor. The following equations were used in the correlation:

1. Vector sum of the measured voltages:

$S=\sqrt{U_{p 1}^{2}+U_{p 2}^{2}+U_{p 3}^{2}}$,

where $U_{p 1}, U_{p 2}, U_{p 3}$ are the voltage measurements from three EUT positions.

2. Field factor $(\sqrt{ } \Omega / \mathrm{m})$ :

$e_{0 y}=\frac{4}{a} \sqrt{Z_{C}}$.

$\sum_{m=1,3,5}^{\infty}\left(\frac{\cosh (M y)}{\sinh (M h)} \cdot \cos (M x) \cdot \sin \left(M \frac{a}{2}\right) \cdot J_{0}(M g)\right)$

where: $a$ is the cell width at $z$ coordinate, $h$ is the septum height at $z$ coordinate, $g$ is the gap width at $z$ coordinate, $x, y, z$ are the coordinates of the EUT centre, $J_{0}$ is the zeroorder Bessel function.

The factor $M$ is calculated as

$M=m \frac{\pi}{a}, m=1,3,5, \ldots, \infty$.

3. Total radiated power $P_{0}$ : $P_{0}=\frac{\eta_{0}}{3 \pi} \cdot \frac{k_{0}^{2}}{e_{0_{y}}^{2} Z_{C}} \cdot S^{2}$,

where: $k_{0}=\frac{2 \pi}{\lambda}$ is the wave number, $\eta_{0}=\sqrt{\frac{\mu_{0}}{\varepsilon_{0}}}$ is the free space wave impedance, $Z_{C}$ is the characteristic impedance of the TEM waveguide, $e_{0_{y}}$ is the TEM mode field factor at the EUT location.

4. Geometry factor $g_{\max }$ :

$g_{\text {max }}=\left\{\begin{array}{l}\left|\frac{1}{r_{1} r_{2}} \sqrt{r_{2}^{2}+r_{1}^{2}-2 r_{1} r_{2} \cos \left[k_{0}\left(r_{2}-r_{1}\right)\right]}\right|_{\text {max }}, \\ \left|\frac{s^{2}}{r_{1}^{3} r_{2}^{3}} \sqrt{r_{2}^{6}+r_{1}^{6}-2 r_{1}^{3} r_{2}^{3} \cos \left[k_{0}\left(r_{2}-r_{1}\right)\right]}\right|_{\text {max }},\end{array}\right.$,

where: $r_{1}=\sqrt{s^{2}+\left(R_{H}-h_{g}\right)^{2}}$ is the distance from the EUT to the receiving antenna, $r_{2}=\sqrt{s^{2}+\left(R_{H}+h_{g}\right)^{2}}$ is the distance of the image of the EUT to the receiving 
antenna, $S$ is the horizontal distance from the EUT to the receiving antenna, $h_{g}$ is the height of the EUT above the ground plane, $R_{H}$ is the height of the receiving antenna above the ground plane.

5. Estimated maximum field strength $(\mathrm{V} / \mathrm{m})$ :

$E_{\text {max }}=g_{\text {max }} \cdot \sqrt{\frac{3 \eta_{0}}{4 \pi} P_{0}}$,

where: $g_{\max }$ is the maximum geometry factor, $\eta_{0}$ is the free-space impedance, $P_{0}$ is the total radiated power of the EUT.

The EMC generic emission standards IEC 61000-6-3 [7] (commercial) and the IEC 61000-6-4 [8] (industrial) state that radiated emissions from EUT should be measured in bands $\mathrm{C}$ $(30 \ldots 300 \mathrm{MHz})$ and $\mathrm{D}(300 \ldots 1000 \mathrm{MHz})$ [9]. To obtain a complete overview of the emissions, both $\mathrm{C}$ and $\mathrm{D}$ bands were included in a single test with start and stop frequencies 30 $\mathrm{MHz}$ and $1 \mathrm{GHz}$, respectively. Measuring bandwidth of 120 $\mathrm{kHz}$ with a $6 \mathrm{~dB}$ EMI filter was used as per requirements stated in the measurement standard CISPR 16-1-1 [9].

Due to the amount of cables in the GTEM cell, the DC-DC converter was considered to be large EUT, but since specific reference emission sources were not available, correction factors were not used in the calculation of the electric field. This may have an impact on the accuracy of the absolute value of the correlated OATS electric field data, but it does not affect the comparability of the results from different operation modes of the same EUT under the same test conditions.

Since data was to be collected only for comparing different operating modes of the DC-DC power converter, rather than for comparing against the standard limits, an average detector was used instead of a quasi-peak detector for faster results.

In total, 11 test series were performed on the DC-DC power converter: 10 different modulation methods (from Method $A$ to Method $J$ ) at nominal load and one test without load using the same modulation method as in the first test.

Measured voltages at the RF port of the GTEM cell of the modulation method $\mathrm{C}$ at three orthogonal EUT orientations are shown in Figure 8

Results from the frequency spectrum analysis in the range of 30...300 MHz from Method E and Method C in Figure 9 show that at rated load, the modulation Method $E$ had the highest maximum radiated emission level $(58.4 \mathrm{~dB} \mu \mathrm{V} / \mathrm{m})$ and the modulation method C (PWM Control with Shifted ShootThrough in One Leg) yielded the lowest level ( 53.9 $\mathrm{dB} \mu \mathrm{V} / \mathrm{m})$. Comparative emission measurement results of modulation Methods $C$ and $E$ are shown in Figure 8

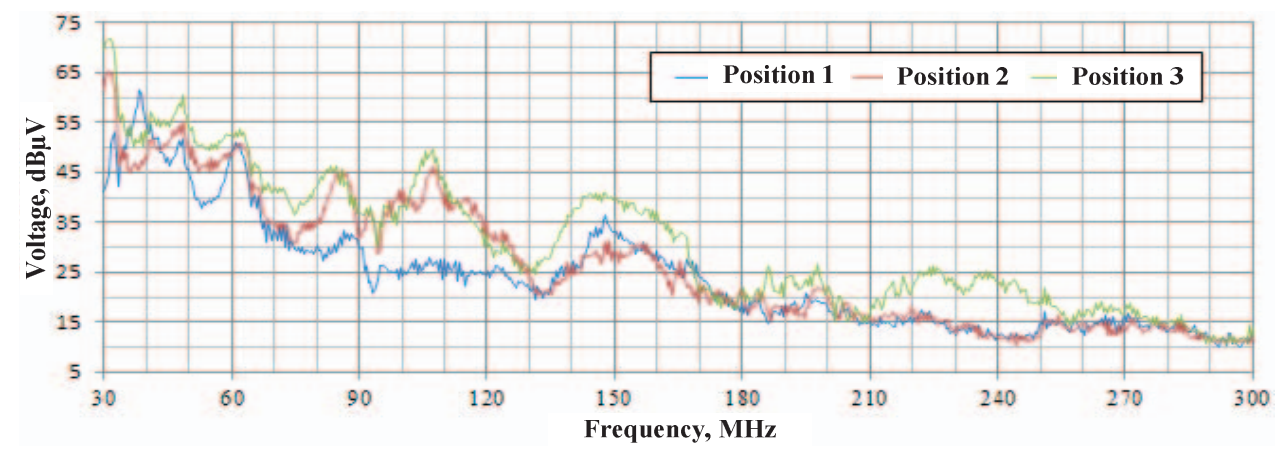

Figure 7 Measured voltages at GTEM cell RF port of the modulation method C at three different EUT orientations

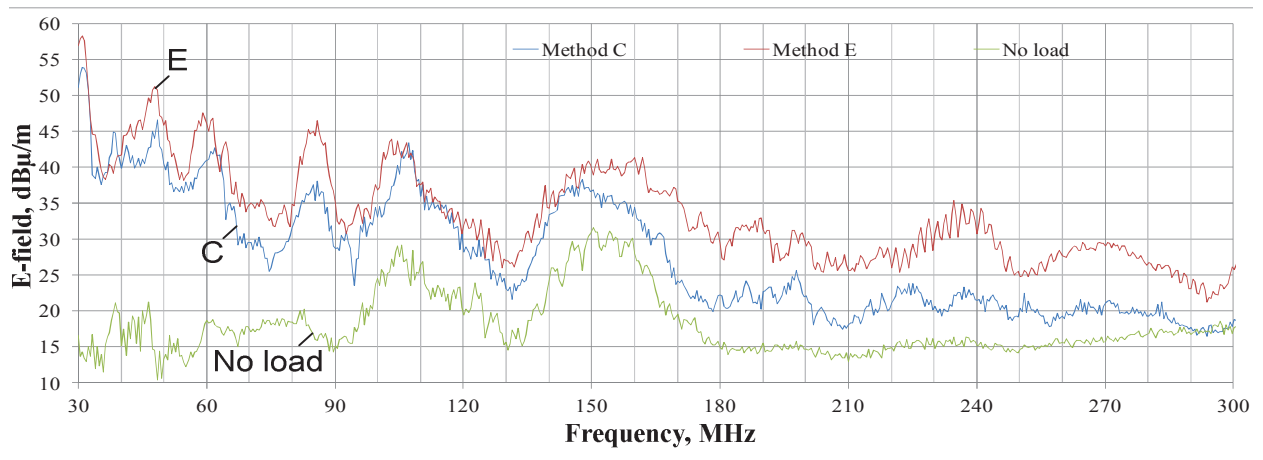

Figure 8 Comparative emission measurement results of modulation methods $\mathrm{C}$ and $\mathrm{E}$. 


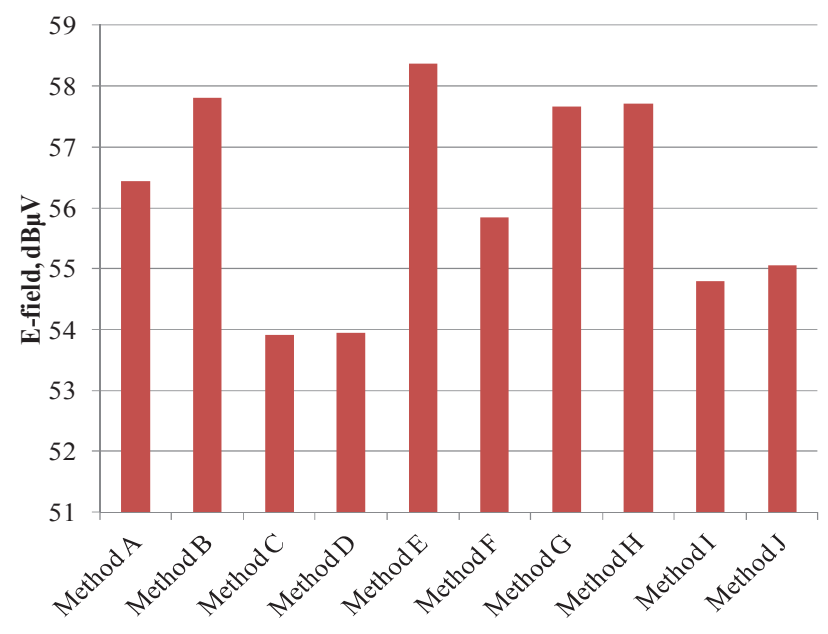

Figure 9 Maximum radiated emission level measurement results.

Results of both modulation Methods $C$ and $D$ were similar at all frequencies (emission level difference $0.03 \mathrm{~dB} \mu \mathrm{V} / \mathrm{m}$ ). Several notable local peaks could be detected at frequencies $30,50,60,85,105$ and $150 \ldots 160 \mathrm{MHz}$. Above $300 \mathrm{MHz}$, the emission levels rapidly decreased to negligible levels.

Frequency spectrum analysis results in the range of 30...300 MHz from Method $E$ and Method $C$ in Figure 8 show that emissions were higher in the lower spectrum area because of the $100 \mathrm{kHz}$ switching frequency of the DC-DC power converter. Modulation Method E test results show an increase not only in the absolute maximum, but also across the whole spectrum. Figure 8 also shows the comparative emission results of the functioning converter without load. At no load conditions, the transistor driver circuits along with isolated DC-DC power supplies and control system contributed to peak emissions at two main frequencies: 105 $(\sim 29.1 \mathrm{~dB} \mu \mathrm{V} / \mathrm{m})$ and $150 \ldots 160 \mathrm{MHz}(\sim 31.5 \mathrm{~dB} \mu \mathrm{V} / \mathrm{m})$.

To further reduce EMI emission, a novel CM voltage compensator can be integrated to the DC-DC converter power circuit [10].

\section{CONCLUSIONS}

Different modulation methods of isolated DC-DC converters with a Quasi Z-source inverter not only affect the voltage boost abilities, efficiency and stability of a converter but also have direct impact on the radiated EMI. The modulation Method $E$ had the highest maximum radiated emission level $(58.4 \mathrm{~dB} \mu \mathrm{V} / \mathrm{m})$ and the Method $C$ had the lowest level $(\sim 53.9 \mathrm{~dB} \mu \mathrm{V} / \mathrm{m})$. Local peaks were detected at frequencies 30, 50, 60, 85, 105 and $150 \ldots 160 \mathrm{MHz}$.

According to the study, Methods $C$ and $D$ were found to be with the lowest emission, which was expected result from the previous analysis.

High emission modulation methods were: $E, B, G$ and $H$. In the case of Method $H$ actually lower emission was expected. Apparently, the number of transistors conducting during the shoot-through has an affect on the emission. The less the better.
As a next step, tests to estimate conducted emission are planned.

\section{ACKNOWLEDGMENTS}

Authors thank Estonian Science Foundation (Project SF0140016s11) (Grant No. 9350), Ubik Solutions and Latvian Council of Science (Grant 416/2012) for financial and material support of this study.

\section{REFERENCES}

[1]

F. Z. Peng, "Z-Source Inverter" IEEE Trans. Ind. Applicat., Volume: 39, Issue: 2, pp.504-510, March 2003.

Vinnikov, D.; Roasto, I., "Quasi-Z-Source-Based Isolated DC-DC Converters for Distributed Power Generation," Industrial Electronics, IEEE Transactions on , vol.58, no.1, pp.192,201, Jan. 2011.

Honnyong Cha; Fang Zheng Peng; Dong-Wook Yoo, "Distributed Impedance Network (Z-Network) DC-DC Converter," Power Electronics, IEEE Transactions on , vol.25, no.11, pp.2722,2733, Nov. 2010.

[4] Roasto, I.; Vinnikov, D.; Zakis, J.; Husev, O., "New Shoot-Through Control Methods for qZSI-Based DC-DC Converters," IEEE Trans. on Industrial Informatics, vol.9, no.2, pp.640-647, May 2013.

[5] I. Roasto, D. Vinnikov, T. Jalakas, J. Zakis, and S. Ott, "Experimental study of shoot-through control methods for qZSI-based DC-DC converters," in SPEEDAM, 2010, pp. 29-34.

[6] IEC 61000-4-20; Electromagnetic compatibility (EMC) - Part 4-20: Testing and measurement techniques Emission and immunity testing in transverse electromagnetic (TEM) waveguides.

[7] IEC 61000-6-3; Electromagnetic compatibility (EMC) Part 6-3: Generic standards Emission standard for residential, commercial and light-industrial environments.

[8] IEC 61000-6-4; Electromagnetic compatibility (EMC) Part 6-4: Generic standards Emission standard for industrial environments.

[9] Comité International Spécial des Perturbations Radioélectriques (CISPR) 16-1-1; Specification for radio disturbance and immunity measuring apparatus and methods - Part 1-1: Radio disturbance and immunity measuring apparatus - Measuring apparatus.

[10] Smolenski, R.; Jarnut, M.; Bojarski, J.; Blinov, A.; Vinnikov, D., "CM voltage compensator for DC/DC converters," Compatibility and Power Electronics (CPE), 2013 8th International Conference on , vol., no., pp.264,268, 5-7 June 2013 